Eur J Clin Chem Clin Biochem

1995; 33:831-837

(c) 1995 Walter de Gruyter \& Co. Berlin - New York

\title{
Predictive Value of Urinary Neopterin in Patients with Lung Cancer
}

\author{
By Peter Kronberger ${ }^{1}$, Günter Weiss ${ }^{2}$, Jörg Tschmelitsch ${ }^{1}$, Dietmar Fuchs ${ }^{2}$, Georg M. Salzer ${ }^{1}$, \\ Helnut Wachter ${ }^{2}$ and Gilbert Reibnegger ${ }^{3}$ \\ 1 Department of Surgery II, University of Innsbruck, Innsbruck, Austria \\ 2 Institute of Medical Chemistry and Biochemistry, and Ludwig-Boltzmann Institute for AIDS Research, \\ University of Innsbruck, Innsbruck, Austria \\ ${ }^{3}$ Institute of Medical Chemistry and Pregl Laboratory, University of Graz, Graz, Austria
}

(Received April 24/July 6, 1995)

Dedicated to Professor Dr. Erich Kaiser on the occasion of his 70th birthday

Summary: Concentrations of neopterin, which is produced by human monocytes/macrophages when stimulated by $\gamma$-interferon, were measured in urine specimens from 72 patients with lung cancer at diagnosis. Other routine clinical and laboratory variables were concomitantly determined. Neither neopterin nor any other laboratory variable studied showed a significant correlation with clinical indicators of the disease (morphologic type, tumour stage, grading, lymph node status, presence of distant metastases). The cancer patients were followed up for up to 10 years, and the abilities of all variables to predict fatal outcome were assessed. In univariate survival analyses, all clinical indicators except morphologic type $(P=0.86)$ were significant predictors of survival $(P<0.002)$, but of all the laboratory variables studied, only neopterin was significantly predictive $(P=0.0013)$. By multivariate survival analysis, a combination of four variables was found to jointly predict survival: lymph node status $(P=0.003$, multivariate model), tumour stage $(P=0.0006)$, grading $(P=0.0047)$ and neopterin $(P=0.0047)$. The data suggest that certain aspects of immune activation may have adverse consequences for the prognosis of patients with lung cancer.

\section{Introduction}

Neopterin is biosynthesized from guanosine triphosphate (GTP). The key enzyme of pteridine biosynthesis, GTP cyclohydrolase I (EC 3.5.4.16), is constitutively present in neural and hepatic tissues but a cytokine-inducible form of this enzyme exists in many other tissues (1). In human monocytes/macrophages, $\gamma$-interferon is the most potent inducer of the enzyme (2), while tumour necrosis factor- $\alpha$ and lipopolysaccharide potentiate the effects of $\gamma$-interferon (3). Human monocytes/macrophages differ from other human and particularly murine cells (including murine macrophages) in that they produce by far the highest amounts of neopterin after induction, whereas all other cells synthesize equal or higher amounts of biopterin derivatives. This peculiarity of human monocytes/macrophages stems from the fact that in these cells, the activities of the constitutive enzymes, 6-pyruvoyl-tetrahydropterin 2'-reductase (EC 1.1.1.220) and sepiapterin reductase (EC 1.1.1.153), which catalyse subsequent reactions of pteridine biosynthesis, are much lower than in all other cells, while the activity of the inducible GTP cyclohydrolase I is particularly high (1).

Neopterin has been widely used as a sensitive in vivo indicator of the activation state of the cellular immune system in a broad variety of diseases, all of which are characterized by involvement of activation of $T$ lymphocytes and production of $\gamma$-interferon and hence, activation of monocytes/macrophages (4-6). In a variety of malignant diseases, neopterin concentrations in serum and excretion in urine have been found to be significantly higher than in healthy controls, and, more importantly, to be an independent prognostic indicator; ele- 
vated neopterin at the time of diagnosis of malignant disease invariably indicates significantly shorter survival times (7).

Lung cancer presents an interesting challenge for a candidate predictor with an immunological basis. Therefore, in a retrospective analysis of 72 patients with lung cancer, the ability of urinary neopterin measured at time of diagnosis to predict the survival probability was assessed.

\section{Materials and Methods}

\section{Patients}

The study was based on 72 patients with lung cancer who were diagnosed at the Department of Surgery II of the University Hospital of Innsbruck in 1983 and 1984 and for whom complete data records were available. All investigations reported here were performed before initiation of any specific tumour therapy. There were 63 men and 9 women; ages at diagnosis ranged from 40 to 80 years with a median age of 62.5 years and an interquartile range from 55 to 71 years. Squamous cell carcinoma was found in 32 patients, 16 had small cell lung cancer, 12 had adenocarcinoma, 9 had large cell carcinoma, and 3 adenosquamous carcinoma. For statistical analyses, the latter 3 patients were combined with those with adenocarcinoma. Stage $T_{1}$ was found in 13 patients, $T_{2}$ in 31 patients, $T_{3}$ in 20 patients, and $T_{4}$ in 8 patients. Stages were pTNM ${ }^{+}$cTNM stages. Lymph node status $\mathrm{N}_{0}$ was seen in 29 patients, 14 had $\mathrm{N}_{1}$, 21 had $N_{2}$, and 8 had $N_{3}$. In 19 patients, the tumour was highly differentiated, 22 patients had moderately differentiated, and 31 poorly differentiated tumour. In 16 patients, distant metastases were present at diagnosis.

Patients were grouped according to their general performance status into three groups: performance status was good for 34 patients, moderate for 25 patients, and poor for 13 patients. Several objective and subjective criteria were used for this classification; all evaluations were made by the same experienced physician.

In 33 patients, therapy consisted of surgery only. Radiation therapy only was administered to 17 patients, and 10 patients were treated by chemotherapy only. Seven patients received surgery and radiation therapy; 3 were treated by surgery and chemotherapy; 1 by chemotherapy and radiation therapy. One patient received surgery, chemotherapy and radiation therapy. Due to the small number of patients in several therapeutic subgroups, therapy was not included in the multivariate analysis of survival.

None of the patients included suffered from accompanying infectious diseases at the time of investigation.

\section{Laboratory examinations}

Neopterin concentrations in first morning urine specimens collected at diagnosis were determined by an optimized and fully automated high performance liquid chromatography technique without oxidative pretreatment. The procedure is described in detail elsewhere (8). By this technique, urinary creatinine is simultaneously determined in the same chromatographic run. In order to compensate for physiologic variations of urine concentrations, neopterin levels are related to these creatinine values and are expressed as $\mu \mathrm{mol}$ of neopterin per mol of creatinine. The native fluorescence of neopterin at $353 \mathrm{~nm}$ excitation wavelength and 438 $\mathrm{nm}$ emission wavelength is used for detection; creatinine is quantitated from its UV absorption at $235 \mathrm{~nm}$. The performance charac-

Tab. 1 Results of laboratory measurements on patients with lung cancer, and their dependence on tumour stage ${ }^{l}$

\begin{tabular}{|c|c|c|c|c|c|c|c|c|c|}
\hline Variable & $\begin{array}{l}\text { First } \\
\text { quartile }\end{array}$ & $\begin{array}{l}\text { Median } \\
\text { value }\end{array}$ & $\begin{array}{l}\text { Third } \\
\text { quartile }\end{array}$ & Range & & $\begin{array}{l}\text { Stage } T_{1} \\
(n=13)\end{array}$ & $\begin{array}{l}\text { Stage } T_{2} \\
(n=31)\end{array}$ & $\begin{array}{l}\text { Stage } T_{3} \\
(n=20)\end{array}$ & $\begin{array}{l}\text { Stage } \bar{T}_{4} \\
(\mathrm{n}=8)\end{array}$ \\
\hline $\begin{array}{l}\text { Neopterin } \\
\quad(\mu \mathrm{mol} / \mathrm{mol} \text { creatinine })\end{array}$ & 174 & 239 & 324 & 99 & -1104 & 220 & 224 & 252 & 289 \\
\hline $\begin{array}{l}\text { Haemoglobin } \\
\quad(\mathrm{g} / \mathrm{l})\end{array}$ & 131 & 141 & 149 & 101 & -175 & 144 & 142 & 139.5 & 141 \\
\hline Haematocrit & 0.40 & 0.41 & 0.45 & 0.35 & 0.50 & 0.44 & 0.43 & 0.42 & 0.42 \\
\hline $\begin{array}{l}\text { Leukocytes } \\
\qquad\left(10^{9} / 1\right)\end{array}$ & 7.2 & 8.45 & 11.25 & $7.7-$ & $-\quad 16.8$ & 7.9 & 8.6 & 8.3 & 8.95 \\
\hline $\begin{array}{l}\text { Thrombocytes } \\
\qquad\left(10^{9} / 1\right)\end{array}$ & 155 & 201.5 & 251.5 & 90 & -507 & 161 & 210 & 196 & 209 \\
\hline $\begin{array}{l}\text { Serum protein } \\
(\mathrm{g} / \mathrm{l})\end{array}$ & 72 & 77 & 82 & 59 & $-\quad 97$ & 77 & 78 & 75 & 82.5 \\
\hline $\begin{array}{l}\text { Urea } \\
\qquad(\mathrm{mmol} / \mathrm{l})\end{array}$ & 4.33 & 5.75 & 7.17 & 2.17 & -15.2 & 5.5 & 5.83 & 6.42 & 4.67 \\
\hline $\begin{array}{l}\text { Aspartate aminotransferase } \\
(\mathrm{U} / \mathrm{l})\end{array}$ & 20 & 25 & 30 & 12 & -144 & 29 & 26 & 22.5 & 25 \\
\hline $\begin{array}{l}\gamma \text {-Glutamyl transferase } \\
(\mathrm{U} / \mathrm{I})\end{array}$ & 15 & 22 & 33 & 7 & $-\quad 99$ & 22 & 24 & 20.5 & 16 \\
\hline $\begin{array}{l}\text { Alkaline phosphatase } \\
\text { (U/I) }\end{array}$ & 108 & 129 & 166 & 29 & -332 & 115 & 125 & 119 & 160 \\
\hline
\end{tabular}

1 Median values of laboratory variables are given. 
teristics of the method were reported earlier (8); briefly, the analytical sensitivity of the method at a peak : noise ratio of $5: 1$ for neopterin is $72 \mathrm{nmol} / \mathrm{l}$ of undiluted urine, or $120 \mathrm{fmol}$ per injection. The sensitivity for creatinine is $36 \mathrm{pmol}$ per injection. The method is linear up to $40 \mu \mathrm{mol} / /$ for neopterin, and $26 \mathrm{mmol} / \mathrm{l}$ for creatinine. Within-run precision of neopterin per creatinine ratio determination ranges from $3.8 \%$ to $6.5 \%$; and between-day precision, from 2.8 to $7.4 \%$.

Leukocyte and thrombocyte count, haematocrit, haemoglobin concentration, serum protein concentration, serum urea concentration and serum concentration of alkaline phosphatase, $\gamma$-glutamyl transferase and aspartate aminotransferase at diagnosis were determined by routine techniques.

\section{Statistical procedures}

Correlations between continuously coded variables were assessed by the non-parametric Spearman's rank correlation technique, since the distributions of observed values were generally nonGaussian. Differences of distributions of laboratory variables among patient groups differing by clinical variables (such as tumour stage) were tested for significance by a non-parametric analysis of variance (Kruskal-Wallis test). Univariate survival analyses were performed using the product-limit model (9); differences between survival curves were assessed for significance by the generalized Savage test (Mantel-Cox test statistic). Categorization of patients according to continuously coded laboratory variables was invariably based on the quartile points of the observed distributions of the variables. Multivariate survival analysis was performed by the proportional hazards technique (10), using forward stepping of variables. For this analysis, laboratory variables were dichotomized on the basis of quartiles of the observed distributions. All statistical analyses were done using the BMDP software package (BMDP Statistical Software, Cork, Ireland). Specifically, program modules BMDP2D (for descriptive analysis), BMDP3S (for non-parametric correlation analysis and analysis of variance), BMDP1L (for product-limit calculations) and BMDP2L (for the proportional hazards model) were employed.

\section{Results}

Table 1 reports results of laboratory measurements, together with median values of biochemical analytes in different tumour stages. Notably, by the Kruskal-Wallis test, the tumour stage showed no significant effect on any of the variable, with the exception of serum urea concentration $(\bar{P}=0.065$; borderline significance). Similarly, the effects of other clinical variables such as performance status, morphologic tumour cell type, grading, and presence of metastasis on the laboratory variables were studied, but no interesting or significant effects were detected. These results are therefore not included.

In an analogous fashion, effects on laboratory variables of other important characteristics of the disease (morphology, grading, presence of distant metastases) were assessed (details not shown). Neopterin in particular was not influenced by performance status $(P=0.13)$ morphologic tumour type $(P=0.22)$, grading $(P=0.37)$, tumour stage $(P=0.47)$, and presence of distant metastases $(P=0.73)$. Only the grouping of the patients on the basis of lymph node status had any effect with borderline significance on neopterin $(P=0.079)$. By Spearman's rank correlation analysis, neopterin showed weak correlations with haematocrit $\left(r_{s}=-0.338\right.$, $P=0.0044)$, leukocytes $\left(r_{s}=0.283, P=0.017\right)$, alkaline phosphatase $\left(r_{s}=0.277, P=0.020\right)$ and haemoglobin level $\left(r_{s}=-0.275, P=0.021\right)$.

\section{Univariate analysis of survival}

Table 2 shows product-limit estimates of cumulative survival probabilities for patients, grouped according to clinical and laboratory variables. For these estimates, laboratory variables were grouped according to quartile points of their respective distributions (see tab. 1). Of the clinical variables, all except morphologic type were significant predictors of survival. Of the laboratory variables only neopterin showed significant predictive power, alkaline phosphatase was of borderline significance, while all other laboratory variables showed no correlation at all with survival expectation. Figure 1 shows the computed cumulative survival expectations for the patients, grouped by three clinical variables and

Tab. 2 Predictive significance of clinical and laboratory ${ }^{1}$ variables in patients with lung cancer

\begin{tabular}{llll}
\hline Variable & $\begin{array}{l}\text { Num- } \\
\text { ber of } \\
\text { groups }\end{array}$ & $\begin{array}{l}\text { Test } \\
\text { statistic }\end{array}$ & $\begin{array}{l}\text { P } \\
\text { value }\end{array}$ \\
& 3 & 29.512 & $<0.0001$ \\
\hline General performance status & 4 & 27.133 & $<0.0001$ \\
Lymph node status & 3 & 20.03 & $<0.0001$ \\
Grading & 2 & 19.375 & $<0.0001$ \\
Distant metastases & 4 & 15.86 & 0.0012 \\
Tumour stage & 4 & 1.15 & 0.76 \\
Morphology & 2 & 1.24 & 0.29 \\
& 4 & 15.76 & 0.0013 \\
Neopterin & 4 & 6.44 & 0.092 \\
Alkaline phosphatase & 4 & 4.55 & 0.21 \\
Haematocrit & 4 & 4.4 & 0.22 \\
$\gamma$-Glutamyl transferase & 4 & 2.8 & 0.42 \\
Haemoglobin & 4 & 2.7 & 0.44 \\
Leukocytes & 4 & 0.84 & 0.84 \\
Aspartate aminotransferase & 4 & 0.57 & 0.91 \\
Urea & 4 & 0.52 & 0.92 \\
Thrombocytes & 4 & 0.28 & 0.96 \\
Protein & &
\end{tabular}

1 Laboratory variables were grouped according to the quartile points of observed distributions (see also tab. 1); categorical variables were used without transformation. For the categories thus defined, product-limit estimates of cumulative survival probabilities were constructed.

${ }^{2}$ Generalized Savage test (Mantel-Cox test) for differences between cumulative survival probabilities.

3 Morphology was examined in two ways: (1) four groups (squamous cell carcinoma, adenocarcinoma including adenosquamous carcinoma, large cell carcinoma, small cell lung cancer); (2) two groups (squamous cell carcinoma combined with adeno/ adenosquamous carcinoma, large cell carcinoma combined with small cell lung cancer). 

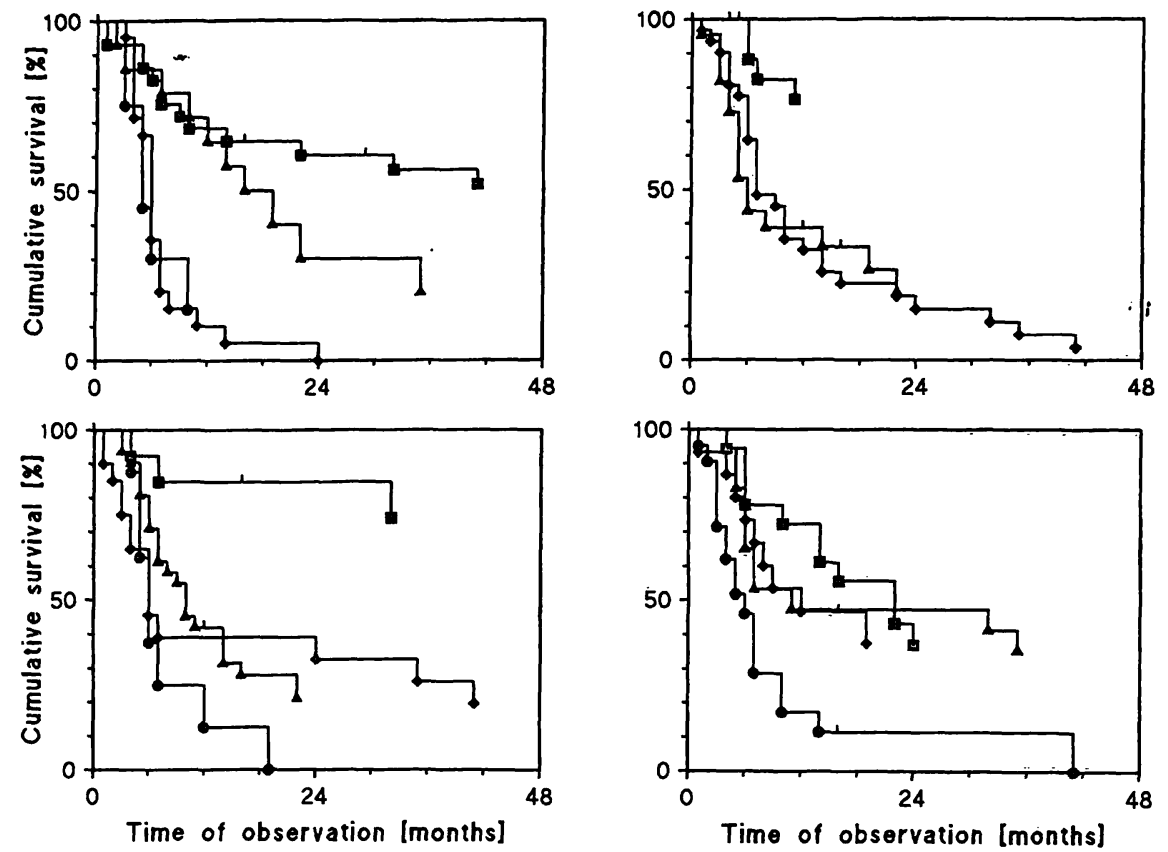

Fig. 1 Product-limit estimates of cumulative survival probabilities. Upper left panel: Patients were stratified by lymph node status (squares, $\mathrm{N}_{0}$; triangles, $\mathrm{N}_{1}$; diamonds, $\mathrm{N}_{2}$; circles, $\mathrm{N}_{3}$ ).

Upper right panel: Patients were stratified by grading (squares, highly differentiated; triangles, moderately differentiated; diamonds, poorly differentiated).

by neopterin concentrations in urine. Notably, no lung cancer-related deaths occurred later than four years after diagnosis. From the survival of the patients grouped by neopterin quartile points, it is obvious that the main difference was noted between the first three, and the last quartile.

\section{Multivariate analysis of survival}

Table 3 shows results of computations using the proportional hazards model. For these analyses, laboratory variables were dichotomized using as cutoff-values either the first (haematocrit, haemoglobin, serum protein: for these variables, declining values are generally associated with deterioration of disease) or the third quartile point of the observed distributions (all other variables, for which increasing values are generally symptomatic for deterioration of disease). Univariate analysis using the proportional hazards model produced essentially the same results as the product-limit approach. When a stepwise regression was performed including all variables as candidate predictors, four variables (lymph node status, tumour stage, grading and neopterin) were found to jointly predict survival.

Figure 2 demonstrates that in patients with lymph node status $\mathrm{N}_{0}$ who according to figure 1 exhibited a long-term survival probability of about $50 \%$, those with neopterin below $324 \mu \mathrm{mol} / \mathrm{mol}$ creatinine had a dramatically better prognosis (long-term survival prob-

ability approximately $30 \%$ ) than those with neopterin exceeding this limit (no patient surviving longer than four years after diagnosis). Similar results demonstrating the independent predictive significance of neopterin were seen in other subgroups of patients (data not shown).

\section{Discussion}

Neopterin is strongly associated with the prognosis of lung cancer; high concentrations of neopterin in urine at the time of diagnosis are a sign for an unfavourable survival expectation, and this predictive information is seen irrespective of other prognostic factors. Thus, neopterin as an indicator of activation of cell-mediated immune mechanism differs from a selection of several routine laboratory variables which carry practically no predictive information. This major result of the present study on lung cancer patients agrees with analogous observations in other malignancies: neopterin was found to be a significant and independent predictor in carcinoma of the uterine cervix (11) or of the ovaries (12), in prostatic carcinoma (13), in patients with hepatocellular carcinoma (14), in various haematological neoplasms $(15-17)$, and in colon caŕcinoma (18).

Our study suffers from the fact that measurements of specific tumour markers were not available. Previous studies, however, investigating the relative predictive 
Tab. 3 Predictive joint significance ${ }^{1}$ of clinical and laboratory variables in patients with lung cancer

\begin{tabular}{|c|c|c|c|c|c|}
\hline Variable & $\begin{array}{l}\text { Cut-off } \\
\text { value }^{2}\end{array}$ & $\begin{array}{l}\text { Univariate } \\
\text { P-value }\end{array}$ & $\begin{array}{l}\text { Regression } \\
\text { coefficient }\end{array}$ & $\begin{array}{l}\text { Standard } \\
\text { error }\end{array}$ & $\begin{array}{l}\text { Multivariate } \\
\text { P-value }{ }^{3}\end{array}$ \\
\hline General performance status & & $<0.0001$ & & & \\
\hline Lymph node status & & $<0.0001$ & 0.531 & 0.143 & 0.0003 \\
\hline Grading & & $<0.0001$ & 0.5448 & 0.203 & 0.0047 \\
\hline Distant metastases & & 0.0002 & & & \\
\hline Tumour stage & & 0.0004 & 0.5997 & 0.174 & 0.0006 \\
\hline Morphology & & 0.31 & & & \\
\hline Neopterin ( $\mu \mathrm{mol} / \mathrm{mol}$ creatinine) & 324 & 0.0006 & 0.9272 & 0.316 & 0.0047 \\
\hline Alkaline phosphatase $(U / 1)$ & 166 & 0.026 & & & \\
\hline Aspartate aminotransferase (U/l) & 30 & 0.54 & & & \\
\hline Haemoglobin $(\mathrm{g} / \mathrm{l})$ & 131 & 0.67 & & & \\
\hline Urea $(\mathrm{mmol} / \mathrm{l})$ & 7.17 & 0.89 & & & \\
\hline Leukocytes $\left(10^{9} / 1\right)$ & 11.25 & 0.89 & & & \\
\hline Protein $(\mathrm{g} / \mathrm{l})$ & 72 & 0.89 & & & \\
\hline$\gamma$-Glutamyl transferase (U/I) & 33 & 0.91 & & & \\
\hline Thrombocytes $\left(10^{9} / 1\right)$ & 251.5 & 0.95 & & & \\
\hline Haematocrit & 0.4 & 0.98 & & & \\
\hline
\end{tabular}

1 Multivariate survival analysis by the proportional hazards technique.

value of concomitantly measured neopterin and tumour markers invariably showed that tumour markers, as compared with neopterin, generally show a stronger relationship to tumour stage but not to prognosis. In particular, in women with ovarian carcinoma at second-look laparotomy, CA-125 concentrations were more strongly correlated than neopterin to the amount of malignant tissue found by the surgical exploration. However, neopterin was an independent predictor of further prognosis while CA-125 failed to yield significant prognostic information in a multivariate analysis (19). Similar results were found in multiple myeloma when neopterin was compared with interleukin-6 (17). Thus, at present it appears that in comparison with specific tumour markers, neopterin as a product of an activated cellular immune system is less strongly correlated to the actual amount of malignant tissue. It appears, however, to be

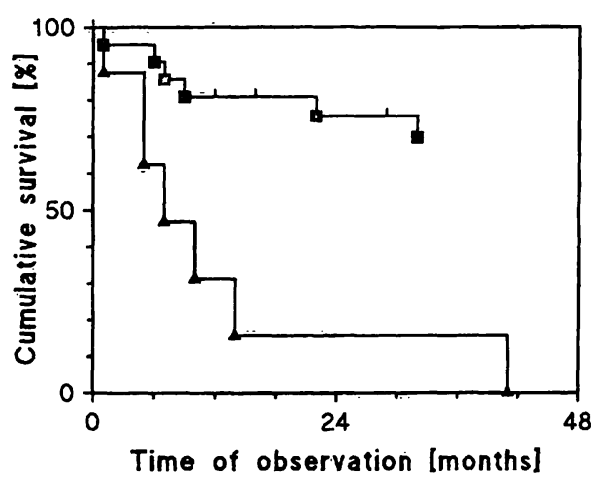

Fig. 2 Product-limit estimates for cumulative survival probabilities for patients with lymph node status $\mathrm{N}_{0}$. Patients were dichotomized by urinary neopterin levels below (squares) versus above (triangles) the third quartile $(324 \mu \mathrm{mol}$ neopterin per mol creatinine).
2 Cut-off value for dichotomizing patients on the basis of laboratory variables.

${ }^{3}$ Only jointly significant variables are included.

superior in providing independent predictive information. We believe that neopterin concentrations reflect important tumour-host interactions rather than the quantity of tumour cells. On the other hand, these interactions are not easily accessible by other variables. The lack of a significant correlation between tumour stage and neopterin in the present study seems to corroborate the foregoing statement. Notwithstanding the predictive significance of neopterin, the well-established indicators of tumour mass and invasiveness were also found to be significant prognostic factors in the present study, and the combination of these with neopterin seems to provide superior information.

Performance status is an important prognostic factor in lung cancer patients. In our study, performance status as a single predictive factor showed the strongest significance of all variables tested. However, in multivariate step-up analyses of survival, performance status entered the model as first variable but was removed later in the stepwise process of variable selection, when other variables entered the model. This was seen regardless of whether the mutually correlated variables, stage and lymph node status, were used for computation, or whether one of them was dropped before the analysis. Therefore, we conclude that performance status, while representing an excellent single predictor, is not an independent prognostic indicator when other predictive variables are taken into consideration.

Our study was based on only 72 patients with lung cancer. Therefore, it is not surprising that the results are not completely in accordance with those of large scale studies. For example, one would expect different sur- 
vival probabilities between stage $T_{2}$ and $T_{3}$ patients, and different survival probabilities between patients with different histologic cell types. Our study is certainly too small to confirm all details of large scale studies; on the contrary, based on previous studies on patients with other types of malignant disease, the main aim of our investigation was the assessment of a possible predictive significance of neopterin concentrations in urine of lung cancer patients.

The consistent observation of raised neopterin production and secretion in many tumour patients, as well as the invariably found correlation between high neopterin levels (and, hence, in vivo activation of cellmediated immune reactions) and poor prognosis in various malignancies, awaits definitive explanation. The data on neopterin levels in various tumour patients seem consistent with the view that in these patients recognition of tumour cells by, and early activation mechanisms of, the immune system are intact, but effector functions are severely suppressed.

Recent observations might offer a novel route to a better understanding of this apparently paradoxical situation:

\section{References}

1. Werner ER, Werner-Felmayer G, Fuchs D, Hausen A, Reibnegger G, Yim JJ, et al. Tetrahydrobiopterin biosynthetic activities in human macrophages, fibroblasts, THP-1, and T24 cells. GTP cyclohydrolase I is stimulated by interferon gamma, and 6-pyruvoyl-tetrahydropterin synthase and sepiapterin reductase are constitutively present. J Biol Chem 1990; 265:3189-92.

2. Huber C, Batchelor JR, Fuchs D, Hausen A, Lang A, Niederwieser $\mathrm{D}$, et al. Immune response-associated production of neopterin. Release from macrophages primarily under control of interferon gamma. J Exp Med 1984; 160:310-6.

3. Werner-Felmayer G, Werner ER, Fuchs D, Hausen A, Reibnegger $\mathrm{G}$, Wachter $\mathrm{H}$. Tumor necrosis factor alpha and lipopolysaccharide enhance interferon-induced tryptophan degradation and pteridine synthesis in human cells. Biol Chem HoppeSeyler 1989; 370:1063-9.

4. Fuchs D, Hausen A, Reibnegger G, Werner ER, Dietrich MP, Wachter $H$. Neopterin as a marker for activated cellular immunity: application in HIV infection. Immunol Today 1988; 9:150-5.

5. Wachter H, Fuchs D, Hausen A, Reibnegger G, Werner ER Neopterin as a marker for activation of cellular immunity: immunological basis and clinical application. Adv Clin Chem 1989; 27:81-141.

6. Wachter H, Fuchs D, Hausen A, Reibnegger G, Weiss G, Werner ER, et al. Neopterin: biochemistry, methods, clinical application. Berlin: Walter de Gruyter, 1992.

7. Reibnegger G, Fuchs D, Fuith LC, Hausen A, Werner ER, Werner-Felmayer $G$, et al. Neopterin as a marker for activated cellmediated immunity: application in malignant disease. Cancer Det Prev 1991; 15:483-9.

8. Fuchs D, Weiss G, Reibnegger G, Wachter H. The role of neopterin as a monitor of cellular immune activation in transplantation, inflammatory, infectious and malignant diseases. Crit Rev Clin Lab Sci 1992; 29:307-41.

9. Kaplan EL, Meier P. Nonparametric estimation from incomplete observations. J Am Stat Assoc 1958; 53:457-81. neopterin enhances reactions mediated by free radicals $(20-22)$. In particular, neopterin strongly enhances luminol-dependent chemiluminescence induced by hydrogen peroxide (in the presence of iron chelator complexes) or by chloramine $T$, and also cytotoxicity of chloramine $\mathrm{T}$ against bacterial cultures. On the other hand, its reduced form, 7,8-dihydroneopterin which is also secreted in excess by activated human monocytes/ macrophages, is a strong scavenger of free radicals (2024). Since activated macrophages gain their cytotoxic potential by their production and secretion of activated oxygen species (oxidative burst), it is tempting to speculate that human macrophages fine-regulate their cytotoxic capacity by means of neopterin and 7,8-dihydroneopterin. At present, research on the interaction between neopterin, and pteridines in general, and free radicals is in its infancy. It is, therefore, too early to draw definitive conclusions. Nevertheless, free radicals have been implicated in the most diverse biological phenomena (25), and future studies on the possible relevance of interactions between pteridines and free radicals would seem highly promising.

10. Cox DR. Regression models and life tables (with discussion). J R Stat Soc 1972; 34:187-220.

11. Reibnegger GJ, Bichler AH, Dapunt O, Fuchs D, Fuith LC, Hausen $A$, et al. Neopterin as a prognostic indicator in patients with carcinoma of the uterine cervix. Cancer Res 1986; 46:950-5.

12. Reibnegger G, Hetzel H, Fuchs D, Fuith LC, Hausen A, Werner ER, et al. Clinical significance of neopterin for prognosis and follow-up in ovarian cancer. Cancer Res 1987; 47:4977-81.

13. Lewenhaupt A, Ekman P, Eneroth P, Erikkson A, Nilsson B, Nordström L. Serum levels of neopterin as related to the prognosis of human prostatic carcinoma. Eur Urol 1986; $12: 422-5$

14. Kawasaki H, Watanabe $H$, Yamada $S$, Watanabe $K$, Suyama A. Prognostic significance of urinary neopterin levels in patients with hepatocellular carcinoma. Tohoku J Exp Med 1988; 155:311-8

15. Abate G, Comella P, Marfella A, Santelli G, Nitsch F, Fiore $M$, et al. Prognostic relevance of urinary neopterin in nonHodgkin's lymphoma. Cancer 1989; 63:484-9.

16. Denz H, Grünewald K, Thaler J, Huber H, Fuchs D, Hausen A, et al. Urinary neopterin as a prognostic marker in haematological neoplasias. Pteridines 1989; 1:167-70.

17. Reibnegger $G$, Krainer $M$, Herold $M$, Ludwig $H$, Wachter $H$, Huber $\mathrm{H}$. Predictive value of interleukin- 6 and neopterin in patients with multiple myeloma. Cancer Res 1991; $51: 6250-3$.

18. Weiss G, Kronberger P, Conrad F, Bodner E, Wachter H, Reibnegger $G$. Neopterin and prognosis in patients with adenocarcinoma of the colon. Cancer Res 1993; 53:260-5.

19. Fuith LC, Dapunt O, Hetzel H, Reibnegger G, Wachter H. Second-look operation in women with ovarian cancer. I. Concentrations of neopterin in urine and CA-125 in serum at secondlook laparotomy. Tumor Diagn Ther 1990; 11:147-51. 
20. Weiss G, Fuchs D, Hausen A, Reibnegger G, Werner ER, Werner-Felmayer $G$, et al. Neopterin modulates toxicity mediated by reactive oxygen and chlorine species. FEBS Lett 1993; 321:89-92.

21. Murr C, Fuchs D, Gössler W, Hausen A, Reibnegger G, Werner $E R$, et al. Enhancement of hydrogen peroxide-induced luminol-dependent chemiluminescence by neopterin depends on the presence of iron chelator complexes. FEBS Lett 1994; 338:223-6.

22. Reibnegger G, Fuchs D, Murr C, Dierich MP, Pfleiderer W, Wachter H. Effects of pteridines on luminol-dependent chemiluminescence induced by chloramine-T. Free Rad Biol Med 1995; 18:515-23.

23. Heales SJR, Blair JA, Meinschad C, Ziegler I. Inhibition of monocyte luminol-dependent chemiluminescence by tetrahydrobiopterin, and the free radical oxidation of tetrahy- drobiopterin, dihydrobiopterin and dihydroneopterin. Cell Biochem Funct 1988; 6:191-5.

24. Kojima S, Icho T, Kajiwara Y, Kubota K. Neopterin as an endogenous antioxidant. FEBS Lett 1992; 304:163-6.

25. Richter C. Pro-oxidants and mitochondrial $\mathrm{Ca}^{2+}$ : their relationship to apoptosis and oncogenesis [minireview]. FEBS Lett 1993; 325:104-7.

Univ.-Prof. Dr. Gilbert Reibnegger

Medizinisch-Chemisches Institut

und Pregl-Laboratorium

Karl-Franzens-Universität

Harrachgasse 21/II

A-8010 Graz

Austria 
